\title{
Interface de Eletrônica e Objeto Interativo como Introdução ao Knowledge Based Design
}

\section{Eletronic Interface and Interactive Object as Knowledge Based Design Introduction}

\author{
Ana Paula Baltazar \\ Universidade Federal de Minas Gerais, Brazil \\ Baltazar.ana@gmail.com \\ José dos Santos Cabral Filho \\ Universidade Federal de Minas Gerais, Brazil \\ cabralfilho@gmail.com \\ Mateus de Souza van Stralen \\ Universidade Federal de Minas Gerais, Brazil \\ Mateus.stralen@gmail.com \\ Guilherme Ferreira de Arruda \\ Universidade Federal de Minas Gerais, Brazil \\ guiarruda.arq@gmail.com
}

\author{
Estevam Quintino Gomes Junior \\ Universidade Federal de Minas Gerais, Brazil \\ estevamgomes@gmail.com \\ Laís Rodrigues Pizano \\ Universidade Federal de Minas Gerais, Brazil \\ laispizano@gmail.com \\ Fabiano Nardy de Moraes \\ Universidade Federal de Minas Gerais, Brazil \\ fabianonardy@gmail.com
}

\begin{abstract}
This paper discusses the evolution of an interface used to introduce eletronics in the foundation design course at the School of Architecture at Universidade Federal de Minas Gerais, Brazil. The use of this interface by the students triggers the discussion of the possibilities of new technologies in architecture and initiates a tactical learning process in which the students are responsible for the very content they are learning. This interface introduces the discussion of interactivity and indeterminism, crucial to the development of the two main works of the course: an individual interactive object and an urban interactive intervention in group.
\end{abstract}

Keywords: Eletronics; Interface; Interactive object; Learning process; Mathetics

\section{Introduction}

Esse artigo apresenta uma das estratégias adotadas na disciplina Fundamentação para Projeto de Arquitetura e Urbanismo I, no primeiro período do curso diurno de arquitetura e urbanismo da Universidade Federal de Minas Gerais para iniciar o aprendizado tático baseado na problematização a partir da introdução de novas tecnologias.

O objetivo da disciplina é sensibilizar os estudantes em quatro aspectos fundamentais na formação do arquiteto: a percepção, a imaginação/proposição, a produção/execução e a representação. Pretende-se romper com o modelo de ensino-aprendizagem incorporado pela maioria dos estudantes que vem do ensino médio das escolas brasileiras, baseado na transferência de conteúdo e controle, por meio de testes prescritos, do conhecimento adquirido. Contra essa lógica, a disciplina trabalha com os estudantes a sua responsabilidade na construção dos conteúdos, ampliando sua possibilidade de autonomia no aprendizado e promovendo o compartilhamento de informação pelos próprios estudantes. Para que os alunos, sem conhecimento ou repertório prévio, sejam capazes de engajar no processo de projeto, adotamos uma abordagem pedagógica fundamentada na matética - termo discutido por Seymour Papert (1980) para definir a arte de aprender em oposição à didática, a arte de ensinar - e o aprendizado par-a-par ( $P 2 P$ learning), introduzindo novas tecnologias que promovem indeterminação e interatividade (de eletrônica simples à computação física). O estudante deve aprender a aprender, despertando sua capacidade de trabalho coletivo, de compartilhamento de informação, seu senso crítico e, principalmente, sua auto-crítica.

A arquitetura é entendida na disciplina como espaço-evento, superando o foco no "objeto" arquitetônico em prol das relações que acontecem no espaço. Sendo assim, o espaço é visto como interface para as relações sociais. Tal espaço-interface pode ser ou prescritivo dos usos e reações das pessoas ou pode ser indeterminado promovendo a interação das pessoas com o próprio espaço e entre elas de maneira dialógica (Flusser, 1999). 0 conceito de interatividade é fundamental nas discussões da disciplina, que informam dois trabalhos práticos: um objeto interativo desenvolvido individualmente e uma intervenção 
urbana interativa, desenvolvida em grupo, que é o ápice do curso. O uso de eletrônica é exigido nesses dois trabalhos ampliando as possibilidades de interação e permitindo discutir o papel das novas tecnologias na arquitetura.

\section{A Interface de Eletrônica}

No âmbito da disciplina a compreensão, concepção e construção de circuitos eletrônicos são um desafio para professores, pesquisadores, monitores e alunos. Muitas vezes, isso acabou por consumir tempo e esforços que poderiam ser direcionados a outras atividades. Para lidar com tal desafio buscou-se facilitar a compreensão do funcionamento de circuitos e criar familiaridade com os componentes eletrônicos por meio de uma espécie de interface composta por placas que ao serem agrupadas montam um circuito eletrônico. Tal interface foi proposta inicialmente como recurso didático para o entendimento de eletrônica, sendo que o professor apresentava as possibilidades para os alunos e estes podiam também experimentar o agrupamento das placas. Posteriormente, como a disciplina foi sendo reformulada e cada vez mais a lógica de "ensino" sendo abandonada e a de "aprendizado" priorizada, a interface também foi reformulada, tanto para viabilizar a abordagem matética quanto para estimular a aprendizagem $P 2 P$.

\section{Primeira versão da interface de eletrônica}

A primeira versão dessa interface, desenvolvida por Mateus Stralen (2009), visava basicamente experimentar diversas relações de inputs e outputs em circuitos eletrônicos simples. Foram confeccionados blocos quadrados de madeira, contendo partes de circuitos e componentes eletrônicos, que poderiam ser encaixados um ao outro para fechar um circuito (figura 1). O primeiro bloco era conectado a uma fonte de $5 \mathrm{~V}$ e servia para alimentar o sistema. Os demais blocos eram divididos em dois grupos: inputs e outputs. O grupo dos inputs era formado por blocos com sensores de input mecânico (botões variados e potenciômetros), sonoro (microfone), luminoso (LDR e infra-vermelho), elétrico (relé). O grupo dos outputs era formado por outputs mecânicos (motor), sonoros (buzzer e alto-falante) e luminosos (leds e lâmpadas de $5 \mathrm{~V})$. Nos blocos, fios pretos e vermelhos deixavam claro o caminho percorrido pela eletricidade entre o pólo positivo e negativo da fonte. O contato entre as duas placas era feito por terminações metálicas. Essa versão foi usada pelos professores em sala de aula como um instrumento didático para demonstração dos conceitos de eletrônica.

O intuito deste sistema não era o de substituir a protoboard, uma plataforma para prototipagem de circuitos mais aberta, adequada para testes de circuitos complexos. A protoboard não deixa claras as ligações entre os componentes e por isso não é muito intuitiva. Assim, a interface de eletrônica acaba sendo mais promissora para o objetivo pedagógico da disciplina, mesmo que a prototipagem de circuitos usando a protoboard seja usada como recurso posteriormente, quando da execução do objeto e da intervenção urbana interativos.
A principal vantagem dessa primeira versão foi promover uma compreensão intuitiva de funcionamento de alguns componentes e conceitos de input e output. O sistema dos blocos tornou possível testar a relação entre inputs e outputs diversos de uma forma intuitiva, através da simples recombinação dos quadrados, desmistificando a aparente complexidade de muitos sistemas eletrônicos. Este sistema se mostrou propício ao desenvolvimento rápido de protótipos de circuitos simples. Contudo, por ser um único kit conectado à fonte de energia alternada, não tinha mobilidade suficiente para que todos os alunos experimentassem livremente e o professor acabava centralizando o "ensino", caindo na lógica de transferência de conhecimento.
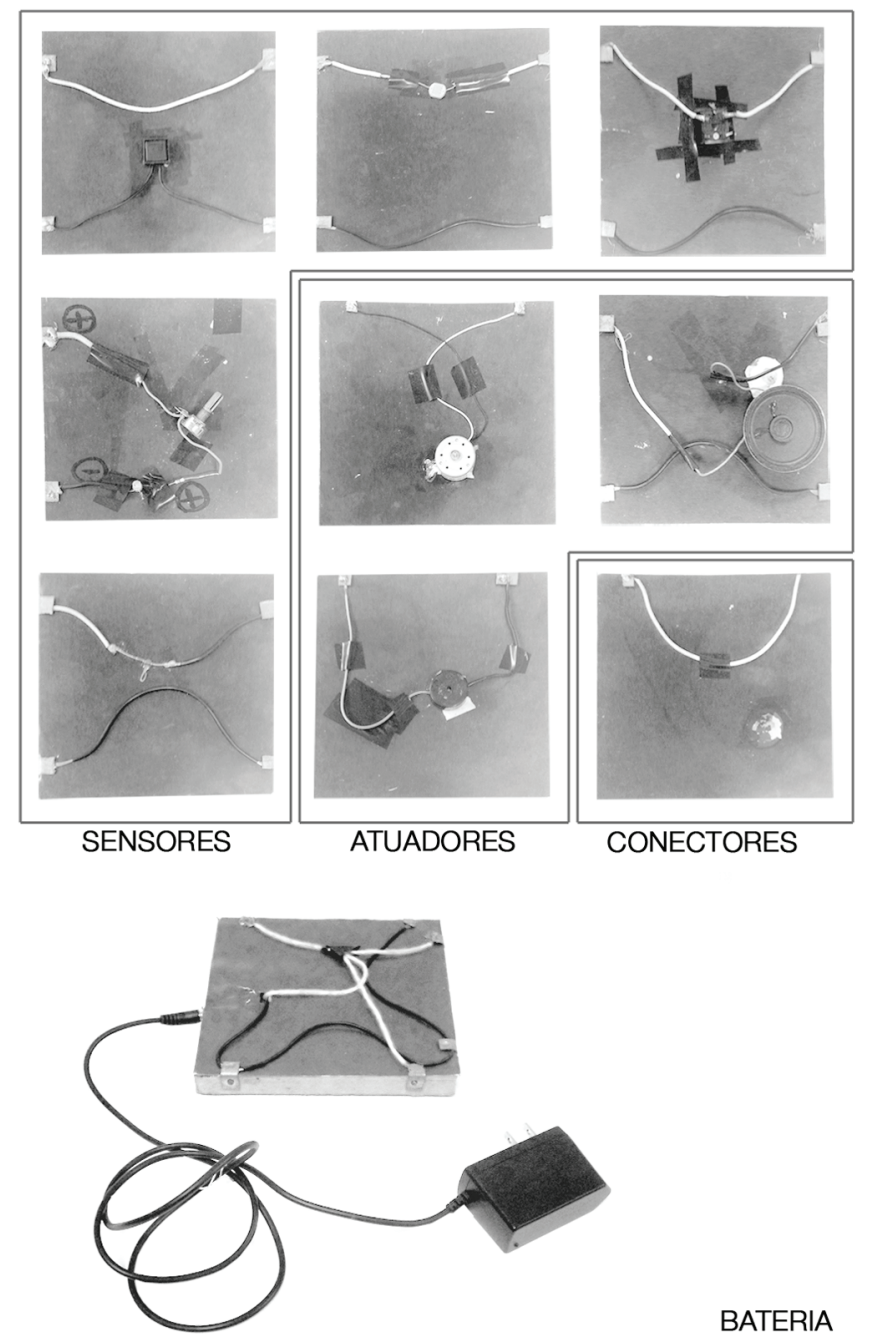

BATERIA

Figura 1: A primeira versão da interface de eletrônica. Acervo LAGEAR (Laboratório Gráfico para Experimentação Arquitetônica).

\section{Segunda versão da interface de eletrônica: multiplicando os kits}

Para que a aprendizagem fosse mais efetiva, baseada na matética, foi necessário uma mudança na abordagem, dando autonomia aos alunos. Seguindo a mesma lógica da primeira versão foram produzidos seis kits de chapas quadradas de papelão paraná com os contatos de papel alumínio (figura 2). Os mesmos inputs e 
outputs foram usados com a adição do transistor nos inputs. A fonte foi trocada por uma bateria de $9 \mathrm{~V}$, eliminando a necessidade de estar próximo a uma fonte de energia, dando mais liberdade de movimentação e construção. Com os seis kits, pudemos experimentar a estratégia matética em que grupos de alunos negociavam entre si a montagem dos circuitos aprendendo, colaborativamente, a partir da própria experiência. A turma foi dividida em grupos de oito alunos e cada grupo recebeu um kit. Os grupos eram solicitados a montar diversos circuitos com o repertório das plaquinhas e discutir possibilidades de automação e interatividade entre as pessoas e das pessoas com objetos e espaços que fizessem uso de componentes eletrônicos. Essa versão manteve o aprendizado intuitivo de circuitos eletrônicos simples e ampliou as possibilidades de engajamento dos alunos no próprio aprendizado, estimulando discussões que ultrapassam a eletrônica, como interatividade e indeterminismo no projeto de objetos e espaços. O principal problema com essa versão foi a precariedade das plaquinhas, principalmente de suas conexões que sempre geravam mal contato e atrapalhavam o desenvolvimento do circuito pretendido pelo grupo.

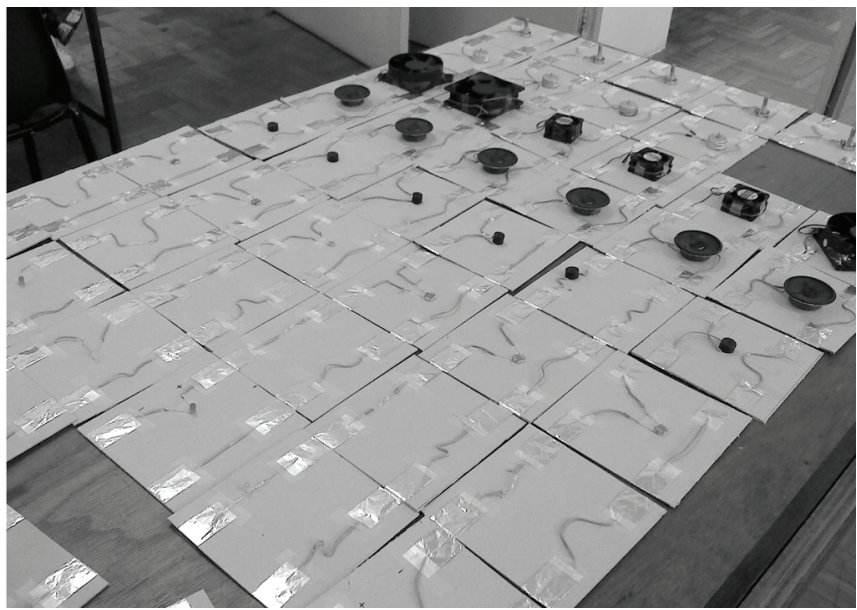

Figura 2: A segunda versão da interface de eletrônica. Acervo LAGEAR (Laboratório Gráfico para Experimentação Arquitetônica).

\section{Terceira versão da interface de eletrônica: espacialização da montagem}

Quando os circuitos apresentavam problemas, não era possível para o aluno distinguir se a causa era a precariedade das placas e dos contatos ou um erro na sua concepção do circuito. Foi desenvolvida, então, uma terceira versão, melhorando as conexões e incorporando também a possibilidade de espacialização da montagem (figura 3). O novo modelo foi elaborado com chapas de madeira de $6 \mathrm{~mm}$, com conexão de fita adesiva de cobre, evitando a necessidade de manutenções constantes. Conta com os mesmos inputs e outputs da versão anterior. Para conectar as peças, foram criados encaixes dentados que permitem uma conexão mais estável, viabilizando a possibilidade da tridimensionalidade nas composições (figura 4). As novas placas têm cinco formas diferentes com encaixes perpendiculares (não coplanares), o que permite uma maior variedade na combinação e na espacialização da montagem. Isso tem funcionado como catalisador para os grupos, que não só se envolvem com as possibilidades de montagem por muito mais tempo, como se empenham mais nas discussões sobre interatividade. Também ficou claro o maior engajamento criativo dos estudantes com essa nova versão da interface.
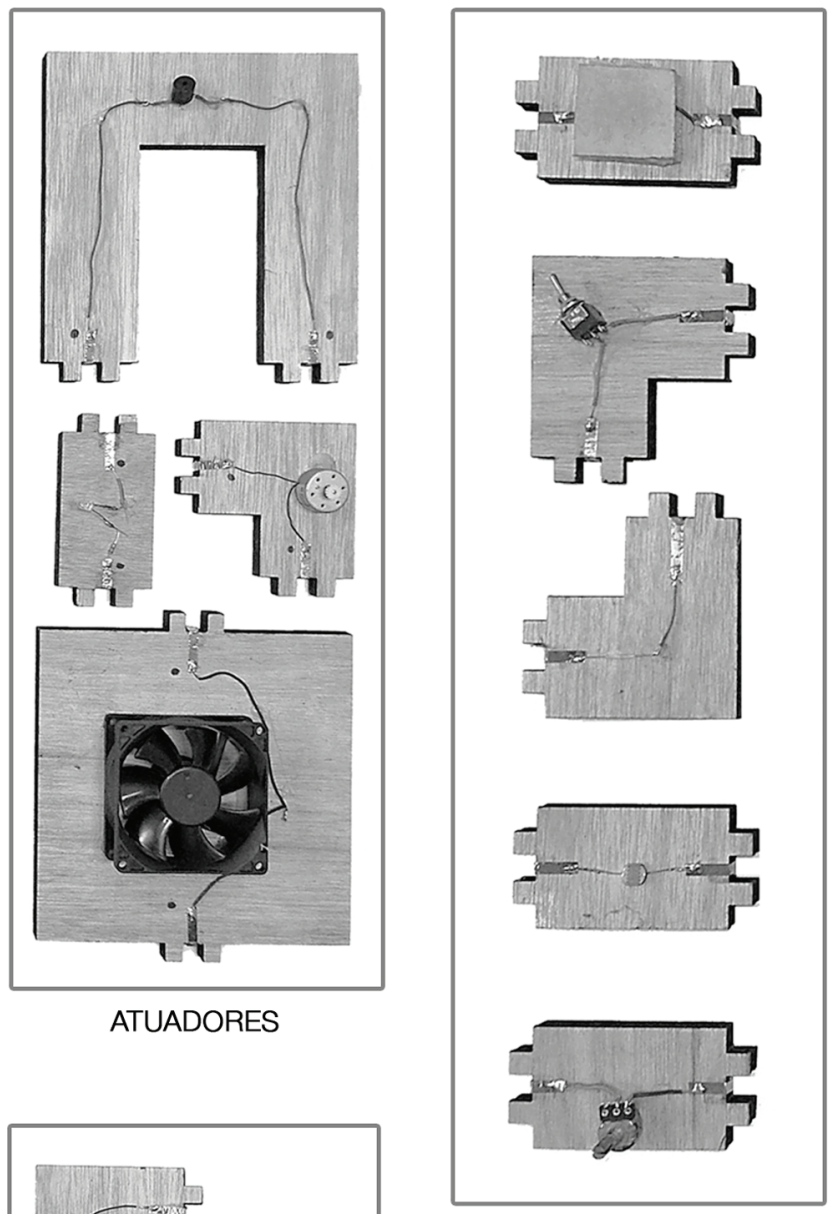

SENSORES

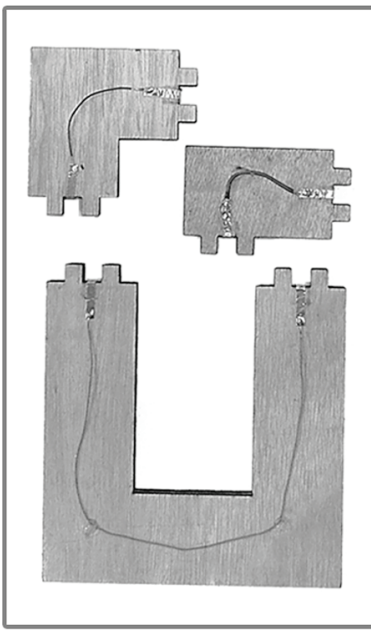

CONECTORES

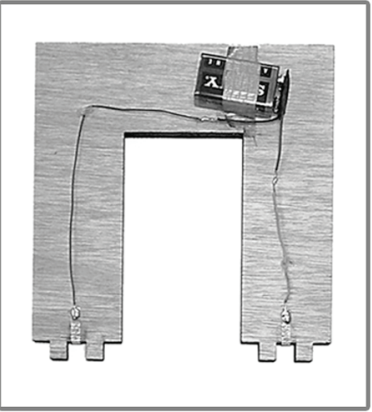

BATERIA
Figura 3: A terceira versão da interface de eletrônica. Acervo LAGEAR (Laboratório Gráfico para Experimentação Arquitetônica). 

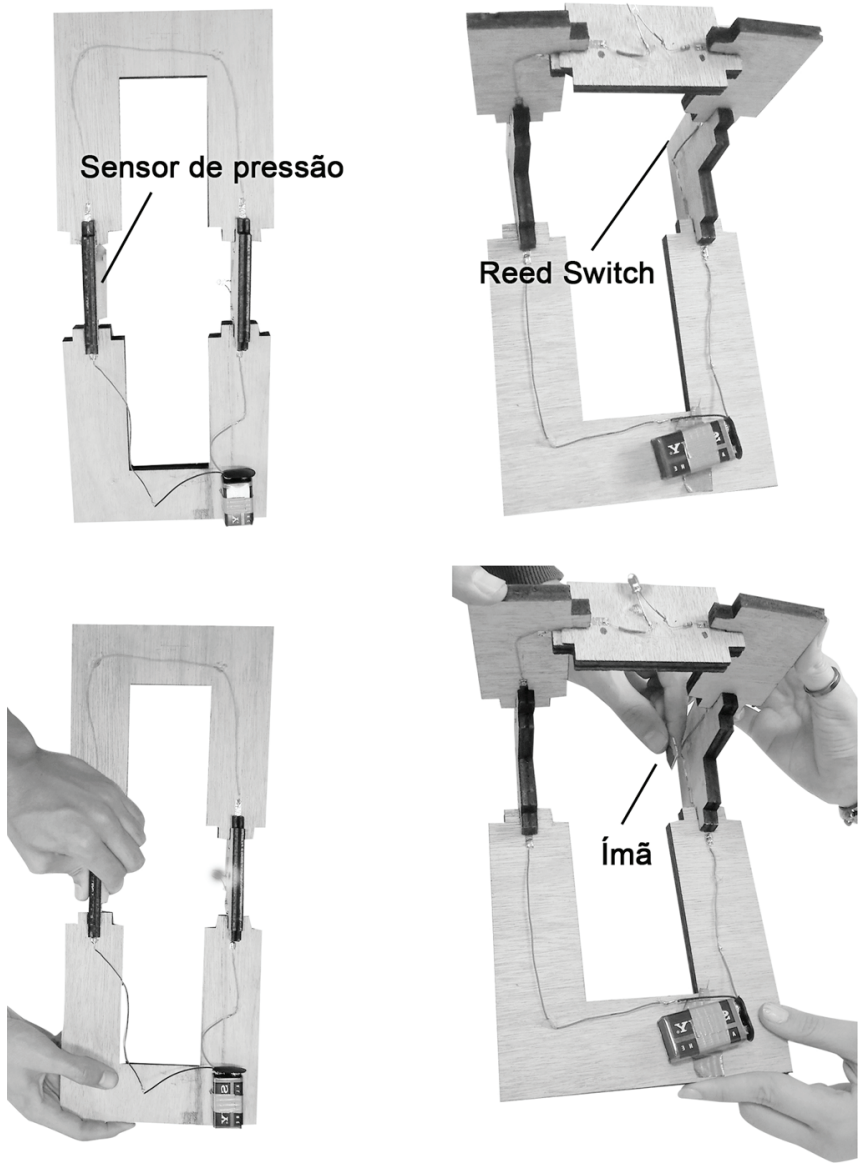

Figura 4: Dois exemplos de circuitos sendo acionados. Acervo LAGEAR (Laboratório Gráfico para Experimentação Arquitetônica).

\section{O Aprendizado $\mathbf{P 2 P}$}

Conforme apresentado acima, a primeira versão da interface de eletrônica seguia a lógica tradicional do modelo de ensino professor-aluno por ser uma plataforma para demonstração dos princípios eletrônicos. A interface não propiciava o envolvimento direto dos alunos na exploração e concepção dos circuitos, principalmente por ser apenas um kit com intuito primordial de apresentação pelo professor.

Já a segunda versão, principalmente por ter sido desenvolvida em uma maior quantidade de kits, parte de princípios da heurística (Papert, 1980), provocando cada grupo de alunos a explorar a interface, no intuito de investigar e problematizar as possibilidades do uso da eletrônica em seus futuros projetos. Essa nova versão pode ser entendida como um "objeto transicional" para a aprendizagem de eletrônica (Papert, 1980). Ela serve como ponte para o entendimento de conceitos abstratos a partir da simplificação e da exteriorização dos circuitos e componentes.

A precariedade da interface é estratégica no processo de desmistificação, que tem por objetivo deslocar o foco para a discussão da interatividade, deixando os alunos bastante à vontade com os princípios de eletrônica. Ela também é aberta, permitindo aos estudantes rearranjarem as mesmas para construção e experimentação. Além disso, o design das placas permite a interação entre os alunos. Contudo a precariedade excessiva acabava desestimulando o aprendizado.

Na terceira versão, a interação entre os alunos permanece pois os encaixes não são autoportantes; os alunos têm que se unir para que os circuitos se fechem. Contudo, os fios e os encaixes são visíveis e o design permite que as plaquinhas sejam autoexplicativas, de maneira que os alunos aprendam por experimentação. Um erro na construção do circuitos estimula à exploração de outras possibilidades uma vez que a ligação entre as placas é mais estável.

Num primeiro uso da interface com os alunos foi proposto que incorporassem à montagem do kit algo que tivessem à mão. Um dos grupos usou um grafite de lapiseira para transmitir energia entre um ponto e outro do circuito, enquanto outro grupo usou o flash do celular para sensibilizar um sensor de luz (LDR) e ativar um buzzer, apitando na frequência que a luz piscava. Ficou claro que mesmo que a interface reformulada mantenha a simplicidade, estimule a colaboração e a espacialização criativa da montagem, ainda carece de abertura para receber outros componentes não previstos, podendo se beneficiar de uma expansão (Resinick et al, 2005).

Para ampliar as possibilidades do aprendizado P2P, além da interface descrita acima, os alunos são solicitados a pesquisar sensores e atuadores divididos em seis grupos: sensores analógicos (potenciômetro linear e logarítmico, LDR - resistor dependente de luz etc.), sensores digitais (botão liga-desliga, reedswitch magnético, sensor de mercúrio etc.), atuadores luminosos e sonoros, motores, componentes complementares (transistor, diodo, relé etc.) e fontes de alimentação diversas (corrente alternada e corrente contínua). Os grupos discutem entre si, apresentando uns para os outros o que pesquisaram, desde o funcionamento e possibilidades de aplicação prática em objetos e espaços cotidianos, até o custo e onde comprar.

\section{O Objeto Interativo como Continuidade da Estratégia Matética}

O objetivo pedagógico da disciplina é especialmente o aprendizado coletivo da crítica e da auto-crítica e a problematização sem prefiguração de soluções. A partir das discussões que emergem com o uso da interface de eletrônica, os alunos são demandados a pensar num objeto como interface que promova a interação entre pessoas. Mais que um objeto reativo, o objeto interativo deve ampliar as possibilidades de diálogo entre as pessoas. No que diz respeito à crítica, começa-se por um brainstorm rápido e cada aluno apresenta uma ideia inicial, que é criticada coletivamente. Em grupo, as primeiras ideias são desconstruídas tendo como critério de análise o tipo de interação que seria promovida entre as pessoas. Os objetos são então repensados para enriquecer a proposta de interatividade. Isso 
permite que cada aluno aprimore seu trabalho e que todos desenvolvam postura crítica a partir de critérios objetivos e propositivos (não interessa dizer "gostei" ou "não gostei", os alunos são incentivados a formular objetivamente as possibilidades e limites dos trabalhos dos colegas que criticam e a apontar caminhos para seu aprimoramento).

No que diz respeito à problematização, os alunos são incentivados a observar a proposta de Cedric Price $(2003,51)$ : "[n]inguém deveria estar interessado no projeto de pontes - deveriam se preocupar em como chegar ao outro lado". Isso é discutido nas análises críticas, quando os alunos são incentivados a exercitar o pensamento abstrato sobre as possibilidades de interação desejadas, antes de prefigurarem o objeto limitando sua interatividade.

\section{Conclusão}

A construção do objeto interativo é um exercício que parece trivial e que poderia ser feito sem eletrônica ou sem a abordagem matética. Contudo, a estratégia pedagógica proposta na disciplina visa o engajamento dos alunos numa postura crítica de aprendizagem construída autonomamente. Para isso faz-se necessária uma estratégia bastante estruturada e a interface de eletrônica certamente cumpre esse papel, principalmente por propiciar a desinibição dos alunos para o processo criativo.

Segundo Resinick et al (2005) "[n]o design de ferramentas para suporte da criatividade, uma das decisões mais importantes é a escolha dos elementos primitivos que os usuários irão manipular. A escolha determina, em grande medida, quais ideias os usuário podem explorar com a ferramenta - e quais ideias permanecem escondidas." A interface de eletrônica pretende estimular a exploração das ideias de interatividade, indeterminismo, processo criativo colaborativo e aprendizagem P2P. Por isso não há necessidade de explicitar o funcionamento interno de cada componente.
A experiência com esse exercício abre caminho para um processo de projeto de espaços baseado no conhecimento, uma vez que os alunos experimentam de forma intensa e aberta a aquisição de repertório estranho à sua prática cotidiana levando a intervenções arquitetônica e urbana interativas. Em suma, a interface de eletrônica, atrelada ao exercício do objeto interativo, introduz os alunos a aprender a aprender, o que lhes será útil ao longo do curso nas diversas situações de projeto.

\section{Agradecimentos}

Os autores agradecem à Fapemig, ao CNPq e à Pró-reitoria de Graduação da UFMG (Programa de Monitoria de Graduação PMG e Programa de Inovação e Qualidade no Ensino de Graduação - PIQEG) pelo financiamento das pesquisas e bolsas que permitiram o desenvolvimento do trabalho. Agradecemos também à contribuição das monitoras Laura Pinho Barbosa, Letícia Cunha Alencar e Júlia Mendes Rocha, que desenvolveram a última versão da interface de eletrônica apresentada.

\section{Referências}

Flusser, V. (1999). Design: obstacle for/to the removal of obstacles, in Flusser, The shape of things: a philosophy of design. London: Reaktion.

Papert, S. (1980). Children, Computers and Powerful Ideas. New York: Basic Books.

Price, C. (2003). The square book. Wiley: London.

Resinick, M., et al. (2005). Design principles for tools to support creative thinking, in National Science Foundation Workshop Report on Creative support tools, Washington: NSF. Retirado de http://www.cs.umd.edu/hcil/CST.

Stralen, M. (2009) Arquitetura amplificada: incorporação de dispositivos tecnológicos digitais à arquitetura. Dissertação de Mestrado em Arquitetura e Urbanismo, desenvolvida no NPGAU, Universidade Federal de Minas Gerais (não publicada). 